\title{
MJMR AIRWAY MANAGEMENT IN A PATIENT WITH AN INCIDENTAL FINDING OF VALLECULAR CYST DURING INDUCTION OF GENERAL ANESTHESIA
}

\author{
lo Camille D. Garcia', Mark Gilbert S. Milallos* \\ ${ }^{1}$ Department of Anesthesiology; Vicente Sotto Memorial Medical Center, Cebu City, Philippines \\ ${ }^{2}$ Department of Health Region VII; Doctor of Science in Nursing Student, College of Nursing, Cebu \\ Normal University, Cebu City, Philippines \\ *Correspondence Author's Email: markmilallos@outlook.com
}

\begin{abstract}
Vallecular cysts are benign retention cysts of the minor salivary glands and the most common site is the lingual surface of epiglottis, which causes distortion to the epiglottis when increased in size and eventually fill the vallecular that can block the laryngeal inlet causing severe respiratory distress. Although not common this is a potentially dangerous condition that causes stridor and this has been associated with sudden airway obstruction resulting to death. This is a case of a 9-day old male neonate referred from General Surgery as a direct to Operating Room case due to imperforate anus. He is full term, delivered normally via vaginal delivery with a birth weight of $3 \mathrm{~kg}$. Upon administration of general anesthesia, the vallecular cyst was initially identified and during the PACU stay of the patient, stridor was noted after extubation, which resulted reintubation to stabilize him. Appropriate referral was made and a confirmed diagnosis of vallecular cyst was made. Patient then underwent marsupialization of the cyst. In conclusion, the presence of vallecular cysts poses a challenge to anesthesiologists especially on incidental findings during the administration of general anesthesia. They must be familiar with the algorithms of care for patients with vallecular cysts and should have necessary skills in identifying signs of distress to avoid unfavorable circumstances and demise.
\end{abstract}

\section{Keywords: Airway Management; Vallecular cyst; General Anesthesia}

\section{INTRODUCTION}

Vallecular cysts are benign retention cysts of the minor salivary glands and the most common site is the lingual surface of epiglottis which causes distortion to the epiglottis when increased in size and eventually fill the vallecular (Parelkar, et al., 2012). This can block the laryngeal inlet causing severe respiratory distress is an unusual presentation (Parelkar, et al., 2012). This is not a common but a potentially dangerous condition that causes stridor and this has been associated with sudden airway obstruction resulting to death due to its anatomical location in neonates; furthermore, it has the potential to excite the retching reflex that includes gagging and vomiting (Gangwar, Kuchhai\& Srivastava, 2016). Nearly $10.5 \%$ of congenital laryngeal cysts are Vallecular cyst and its reported incidence rate is at 5.3 cases in every 100,000 live births (Coban, Bas \& Aydin, 2018). This is an uncommon case with great fatality, thus a case report is presented. Treatment techniques include marsupialization and surgical excision with or without CO2 laser (ZamfirChiru-Anton \& Gheorghe, 2016).

Airway management is of utmost importance for neonates with Vallecular cysts at this poses a risk blocking the airways that can lead to death. This case report describes the medical and anesthetic management and considerations preoperatively, intraoperatively and postoperatively in patients with incidental findings of Vallecular cyst. This could further supplement current literature like published case studies that can be used in other papers and similar cases in the future.

\section{LITERATURE REVIEW}

Vallecular cyst is rare but a well-recognized cause of upper airway obstruction commonly found on newborns and infants (Namshikar, Dukle, \&Sukhthanker, 2016). This can lead to stridor and a complete airway obstruction in infants, which may be triggered by factors like feeding, crying, induction of anesthesia or even 
during awake fibreoptic bronchoscopy (Batra, Kale, \&Malik, 2012). Eventually, this may lead to death among newborns and infants if not diagnosed immediately (Cheng, Ng, Li, \&Hartigan, 2002). The presence of a vallecular cyst poses a challenge for anesthesiologist during induction of general anesthesia and intubation (Namshikar, Dukle, \&Sukhthanker, 2016; Batra, Kale, \&Malik, 2012; \&Cheng, Ng, Li, $\&$ Hartigan, 2002). Maintaining spontaneous respiration during the inhalational induction and preparing for an emergency tracheostomy should be part of the anesthesia plan (Namshikar, Dukle, \&Sukhthanker, 2016; Batra, Kale, \&Malik, 2012; \& Lauro, 2017). Gentle laryngoscopy should be done to prevent cyst rupture and pulmonary aspiration (Namshikar, Dukle, \&Sukhthanker, 2016).

Lauro (2017) stated that early direct laryngoscopy under general anesthesia is advocated by most authorities since airway may be safely secured without patient compromise by inhalation induction with total IV anesthesia maintenance preserving spontaneous ventilation. However, cyst rupture is a risk and this can lead to pulmonary aspiration, loss of airway, patient agitation and forceful inspiration which exacerbate airway collapse, hemorrhage into the cyst from trauma, edema, bleeding. Repeated attempts at intubation should be avoided to minimize risk for rupture (Lauro, 2017). Communication and teamwork with the surgical team is vital to formulate an individualized anesthetic plan (Lauro, 2017).

\section{CASE STUDY}

This is a case of a 9 day old male neonate referred from General Surgery as a direct to Operating Room case due to imperforate anus. He is full term, delivered normally via vaginal delivery with a birth weight of $3 \mathrm{~kg}$. He is temporarily on a nil per os (NPO) status or nothing by mouth.

Upon examining the patient, his weight was $\mathrm{kg}$. His vital signs are stable and patient was afebrile upon examination. He was able to maintain an oxygen desaturation between $96-98 \%$ at room air. Fine rales were noted on thebibasallung fields was noted upon auscultation of chest and lungs. No suprasternal or inspiratory stridor noted. Abdomen was soft and nontender.
Pre-operative assessment was done. Patient was categorized as American Society of Anesthesia (ASA) class 2E.Induction under General Anesthesia was planned using sevoflurane in $100 \%$ oxygen. Atropine of $0.01 \mathrm{mg} / \mathrm{kg}$ was given prior to induction of anesthesia. Direct laryngoscopy was attempted using Macintosh blade 0 and endotracheal tube $3 \mathrm{~mm}$ internal diameter. Upon intubation a cystic swelling approximately $2 \times 2 \mathrm{~cm}$ size was noted at the tip of the blade. However, the decision to push through with endotracheal intubation was done. Placement of endotracheal tube was confirmed. Surgery lasted for 35 minutes and patient was stable throughout surgery. Postoperatively the patient was noted to have stable vital signs and spontaneous breathing. Patient was then extubated fully awake.Upon extubation stridor was noted however vital signs remain stable and no desaturations noted. Patient was then transferred to the Post Anesthesia Care Unit (PACU). Upon Arrival in PACU persistence of stridor was noted. Bradycardia of $100 \mathrm{bpm}$ was noted associated with desaturations to $88 \%$. The decision to reintubate the patient was made.

Patient was further monitored and once stable transferred to Neonatal Intensive Care Unit (NICU).4 days post-surgery patient was referred to the Department of Otorhino-laryngology - Head and Neck Surgery for mass in the upper airway. Patient was then scheduled for direct laryngoscopy.

On the second operation inhalationalanesthesia was done using sevoflurane in $100 \%$ oxygen. ENT surgeon performed laryngoscopy and vallecular cyst was identified. Marsupialization of the cyst was done. By the end of surgery, patient was transported to Pediatric Intensive Care Unit. Patient was monitored every hour and gradual weaning from mechanical ventilation was done. Patient was extubated on the second postoperative day. No stridor or dyspnea noted.

\section{DISCUSSIONS}

Duct cysts represent $75 \%$ of congenital laryngeal cysts and usually arise from the obstruction of the submucosal gland ducts and are mainly found in the vallecular; although, congenitalvallecular cysts are uncommon, they should be included in differential diagnosis of stridor and progressive obstruction of the airway in newborns and infants (Rodríguez, Cuestas\& 
Solernou, 2015).The initial sign of the cyst was the presenting difficulty during intubation in preparation for General Anesthesia and was when the cyst was identified. Direct laryngoscopy under general anesthesia is the major diagnostic tool for confirming the diagnosis of vallecular cyst (Torer, et al, 2015).Thorough airway assessment and planning is mandatory in the anesthetization of patients with vallecular cysts (Kothandan, et al., 2013). The difficulty of intubation was also noted in several case reports.

Anesthetic management of vallecular cyst excision is one of the most challenging procedures encountered in anesthetic practice due to the location of the cyst (Goel, et al., 2018). Furthermore, these patients are at increased risk of airway occlusion resulting in hypoventilation, hypoxemia, or death (Namshikar, Dukle, \& Sukhthanker, 2016). Stridor was noted on the patient after extubation while patient was still in the PACU which is the most common indirect presenting sign of Vallecular cyst. The patient may present with symptoms such as stridor, feeding difficulty, failure to thrive, dysphonia, or respiratory distress(AlAbdulla, 2015). In this case, patient was reintubated after stridor and respiratory distress was noted. Patients may present with acute life threatening airway compromise requiring emergency airway management supporting the need for reintubation (AlAbdulla, 2015). Immediate action is needed since the resulting airway obstruction can lead to serious morbidity and mortality (Gogia, Agarwal,\& Agarwal, 2014). After being noted, patient was referred to ENT (Ears, Nose, and Throat) specialist and was scheduled for direct laryngoscopy. Direct laryngoscopy remains the gold standard for diagnosis, which also helps in detection of laryngomalacia, commonly associated with vallecular cyst (Parelkar, et al., 2012, Aziz, Abdullah,\& Ahmad, 2010, and Mulcahy, et al., 2017). Patient was then scheduled for surgery to address the vallecular cyst. Primary treatment of a vallecular cyst is surgery with a varied recurrence rate, most authors report successful outcomes when marsupialization with or without the use of a CO2 laser (Leibowitz, et al., 2017, Mulcahy, et al., 2017, and Tsai, et al., 2013).

Maintaining a clear airway has always been vital in the management, of patients with vallecular cysts as this can lead to airway obstructions that can result serious morbidity and mortality (Gogia, Agarwal,\&Agarwal, 2014).Methods for preparing and executing proper airway management in a newborn or infant who with apnea and stridor should be always considered (Oelsen, et al., 2018). Physical Exam is still vital in patients presenting noisy breath sounds like noting the characteristics of the breath sounds (Nolder \& Richter, 2015). Stridor is high-pitched sound that can result from airway obstruction at the level of the supraglottis, glottis, subglottis, or trachea (Nolder\&Richter, 2015). In this case, fine rales were noted prior to the induction of anesthesia and upon intubation, a cystic mass was noted that caused some resistance and prompted a change of a smaller tube size. Stridor, labored breathing and desaturations were already evident in the patient immediately after extubation. This prompted reintubation that provided relief to the patient while in the Post Anesthesia Care Unit. Visualization of the airway through laryngoscopy can confirm any blockage in the airway including vallecular cysts (Torer, et al., 2017, andNolder\& Richter, 2015). Aspiration of vallecular cysts may offer quick relied in upper airway obstruction but is not recommended because of the high recurrence rate (Torer, et al., 2017). Direct laryngoscopy again is highly advised for the diagnostic confirmation of vallecular cysts (Parelkar, et al., 2012, Torer, et al., 2017, Aziz, Abdullah, \& Ahmad, 2010, and Mulcahy, et al., 2017); furthermore, marsupialization is the surgery of choice in treating such (Torer, et al., 2017, Leibowitz, et al., 2017, Mulcahy, et al., 2017, and Tsai, et al., 2013) which was also done the patient.

\section{CONCLUSION}

Incidental findings of vallecular cyst can post a challenge to anesthesiologists especially during the intubation process, as the cyst serves as an obstruction on the airway as well on insertion of the endotracheal tube. Anesthesiologists must have keen eye on assessing patients once or resistance is felt on the airway during intubation. A thorough assessment in needed throughout the entire perioperative process. Induction of anesthesia can again a challenge that before intubation, the airway must be visualized to determine patency and appropriated of the tube size. Careful monitoring must be done intra-operatively and post operatively, it is necessary to be quick on 
identifying respiratory distress so that immediate intervention can be provided like reintubation. Vallecular cysts are uncommon cases among pediatric patients and newborn and unusual resistance must not usually present and assessing patients thoroughly to determine the cause so appropriate referral can be made. Indirect symptoms of vallecular cysts like respiratory distress or stridor must be identified while patients are still recovering from the Post Anesthesia Care Unit in order that appropriate measures and referral can be done.

Overall, the presence of vallecular cysts poses a challenge to anesthesiologists especially on incidental findings during the administration of general anesthesia. They must be familiar with the algorithms of care for patients with vallecular cysts and should have necessary skills in identifying signs of distress to avoid unfavorable circumstances and demise.

\section{Conflict of Interest}

The authors declare that they have no conflict of interest.

\section{ACKNOWLEDGEMENT}

The authors would like to acknowledge the Department of Anesthesiology, Training and Research Division, and Medical Records Department of the Vicente Sotto Memorial Medical Center for all the assistance provided.

\section{REFERENCES}

Al Abdulla, A.F. (2015) Congenital Vallecular Cyst Causing Airway Compromise in a 2-Month-Old Girl. Hindawi: Case Reports in Medicine. Retrieved from: https://www.hindawi.com/journals/ crim/2015/975859/

Aziz, A.A., Abdullah, A.F. \& Ahmad, R.A.R.L. (2010) Two Cases of Congenital Vallecular Cyst: A Reminder of the Potentially Fatal Cause of Upper Airway Obstruction in Infants. The Malaysian Journal of Medical Sciences. 17(3) pp: 68-73.

Batra, U. B., Kale, S., \& Malik, A. (2012). Anaesthetic management of an infant with vallecular cyst: $A$ challenging situation. Indian Journal of Anaesthesia, 56(4) pp:423-425.

Cheng, K. S., Ng, J. Li, H., \&Hartigan, P. M. (2002). Vallecular cyst and laryngomalacia in infants:
Report of six cases and airway management. Anesthesia \& Analgesia, 95(5) pp:1248-1250

Coban, K., Bas, C. and Aydin, E. (2018) Vallecular Cyst in a 1 Month Old Infant. Journal of Otolaryngology Research. (1)1.

Gangwar, A., Kuchhai, V., \& Srivastava, P. (2016) A Rare Case of Vallecular Cyst. International Journal of Advanced \& Integrated Medical Sciences. 1(2) pp:88-90.

Goel, N., Bhardwaj, N., Gupta, A, and Gowtham, K. (2018) Giant vallecular cyst excision in infant: Should we proceed without a definite airway? Saudi Journal of Anesthesia. 12(4) pp: 647-649.

Gogia, S., Agarwal, S.K. \& Agarwal, A. (2014) Vallecular cyst in neonates: case series-a clinicosurgical insight, Case Reports in Otolaryngology. 2014(764860)

Kothandan, H., Ho, V.K., Chan, Y.M., \& Wong, T. (2013) Difficult intubation in a patient with vallecular cyst. Singapore Medical Journal; 54(3) pp:e62-e65.

Lauro, H. V. (2017). Congenital cysts involving airway (vallecular cyst, lingual thyroglossal duct cyst). Clinical Pain Advisor. Retrieved from https://www. clinicalpainadvisor.com/home/decision-supportin-medicine/anesthesiology/congenital-cystsinvolving-airway-vallecular-cyst-lingualthyroglossal-duct-cyst/

Leibowitz, J.M., Smith, L.P., Cohen, M.A., Dunhan, B., Guttenberg, M. and Elden, L.M. (2011) Diagnosis and treatment of pediatric vallecular cysts and pseudocysts. International Journal of Pediatric Otorhinolaryngology, 75(7) pp:899-904.

Mulcahy, CF, Reddy, SK, Wikner, EE \& Mudd, PA. (2017) Neonatal airway anomaly: vallecular cyst. BMJ Case Reports. Retrieved from: https:// casereports.bmj.com/content/2017/bcr-2017223082

Namshikar, V. N., Dukle, N. V., \& Sukhthanker, D. S. (2016). Anesthetic management of vallecular cyst excision in an infant: An airway challenge. Saudi Journal of Anaesthesia, 10(3) pp:356-358.

Namshikar, V.N., Dukle, N.V. \& Sukhthanker, D.S. (2016) Anesthetic management of vallecular cyst 
excision in an infant: An airway challenge. Saudi Journal of Anaesthesia. 10(3) pp:356-358.

Nolder, A.R. \& Richter, G.T. (2015) The infant with noisy breathing. Current Treatment Options in Pediatrics, 1(3) pp:224-233.

Oelsen, J.M., Hewett, K.M., Discolo, C.M., \& Jackson, B.F. (2018) Congenital vallecular cyst as a cause of neonatal stridor and apnea. Pediatric Emergency Care. 34(8) pp:152-154.

Parelkar, S.V., Patel, J.L., Sanghvi, B.V., Joshi, P.B., Sahoo, S.K., Sampat, N., Oak, S.N., \& Sathe, N. (2012) An Unusual Presentation of Vallecular Cyst with near Fatal Respiratory Distress and Management Using Conventional Laparoscopic Instruments. Journal of Surgical Technique \& Case Report. 4(2) pp:118-120.

Rodríguez, H., Cuestas, G. \& Solernou, V. (2015) Quiste de valléculacongénitoen un lactante. Acta
Otorrinolaringologica Española; 66(1) pp: 59-60.

Torer, B., Cetinkaya, B., Yilmaz, S., Yilmazer, C., Gulcan, H. (2015) Upper Airway Obstruction in a Newborn with Vallecular Cyst. Journal of Neonatal Surgery. 4(4) pp:45.

Torres, O., Tena, N.M., Murray, B., Sarkar, A. (2017) Novel starch based emulsion gels and emulsion microgel particles: Design, structure and rheology, Eselvier; 178(15) pp:86-94

Tsai, Y.T., Lee, L.A., Fang, T.J., \& Li, H.Y. (2013) Treatment of vallecular cysts in infants with and without coexisting laryngomalacia using endoscopic laser marsupialization: fifteen-year experience at a single-center. International Journal of Pediatric Otorhinolaryngoly, 77(3) pp:424-428.

Zamfir-Chiru-Anton, A. \& Gheorghe, D.C. (2016) Vallecular cysts in clinical practice: report of two cases. Journal of Medicine and Life, 9(3) pp:288-290. 